\title{
Label-free Detection of Human Prostate-Specific Antigen (hPSA) Using Film Bulk Acoustic Resonators (FBARs)
}

Xiubo Zhao ${ }^{1 *}$, Fang Pan $^{2}$, Gregory M. Ashley ${ }^{3}$, Luis Garcia-Gancedo ${ }^{4}$, Jikui Luo ${ }^{3}$, Andrew J. Flewitt ${ }^{4}$, W.I. Milne ${ }^{4}$, Jian R. Lu ${ }^{2 *}$

${ }^{1}$ Department of Chemical and Biological Engineering, University of Sheffield, Mappin Street, Sheffield, S1 3JD, UK

${ }^{2}$ Biological Physics Group, School of Physics \& Astronomy, University of Manchester, Oxford Road, Manchester M13 9PL, UK

${ }^{3}$ Institute for Materials Research and Innovation (IMRI), University of Bolton, Deane Road, Bolton BL3 $5 \mathrm{AB}, \mathrm{UK}$

${ }^{4}$ Electrical Engineering Division, University of Cambridge, JJ Thomson Avenue, Cambridge CB3 0FA, UK

*Corresponding authors at: Department of Chemical and Biological Engineering, University of Sheffield, Mappin Street, Sheffield S1 3JD, UK (X. Zhao); Biological Physics Group, School of Physics and Astronomy, University of Manchester, Oxford Road, Manchester, M13 9PL, UK (J.R. Lu).

E-mails: Xiubo.Zhao@sheffield.ac.uk (X. Zhao); J.Lu@manchester.ac.uk (J. R. Lu) 


\begin{abstract}
Label-free detection of cancer biomarkers using low cost biosensors has promising applications in clinical diagnostics. In this work, ZnO-based thin film bulk acoustic wave resonators (FBARs) with resonant frequency of $\sim 1.5 \mathrm{GHz}$ and mass sensitivity of $0.015 \mathrm{mg} / \mathrm{m}^{2}\left(1.5 \mathrm{ng} / \mathrm{cm}^{2}\right)$ have been fabricated for their deployment as biosensors. Mouse monoclonal antibody, anti-human prostatespecific antigen (Anti-hPSA) has been used to bind human prostate-specific antigen (hPSA), a model cancer biomarker used in this study. Ellipsometry was used to characterize and optimise the antibody adsorption and biomarker antigen binding on gold surface. It was found that the best amount of antibody at the gold surface for effective antigen binding is around $1 \mathrm{mg} / \mathrm{m}^{2}$, above or below which resulted in the reduced antigen binding due to either the limited binding sites (below 1 $\mathrm{mg} / \mathrm{m}^{2}$ ) or increased steric effect (above $1 \mathrm{mg} / \mathrm{m}^{2}$ ). The FBAR data were in good agreement with the data obtained from ellipsometry. Antigen binding experiments using FBAR sensors demonstrated that FBARs have the capability to precisely detect antigen binding, thereby making FBARs an attractive low cost alternative to existing cancer diagnostic sensors.
\end{abstract}

Keywords: FBAR, Biosensors, Biomarker, Cancer diagnostics, Monoclonal antibody, Prostatespecific antigen, Protein adsorption

\title{
1. Introduction
}

Biosensors have been widely employed as tools for biotechnological and biomedical applications such as investigating biomolecular interactions and cancer diagnostics.[1] Most of the existing biosensors are optical instruments which are complex and/or expensive, some of them also needing molecular labelling. For this reason, sensitive, label-free and disposable biosensors are particularly promising and have attracted much attention in the last decade.[2, 3]

Acoustic resonators such as quartz crystal microbalance (QCM), surface acoustic wave devices (SAWs) or film bulk acoustic wave resonators (FBARs) are interesting alternatives in biosensing applications due to their high sensitivity, non-invasiveness and relatively low cost. The basic principles of operation of acoustic resonators are simple: acoustic waves with a well-defined resonant frequency $\left(f_{r}\right)$ are generated through the application of a microwave signal to a set of electrodes deposited on a piezoelectric material. The addition of a mass on a sensitive area lowers the resonant frequency. By tracking changes in $f_{r}$, a variation of the mass bound on the sensing area (for example due to adsorption of a biological sample) can be detected as the mass bound on the resonator is related to the change in $f_{r}$. If the mass bound on the resonator is small (lower than $2 \%$ of the total mass of the resonator), there exists a direct relationship between the fundamental 
resonant frequency and the responsively to mass loading: higher frequency devices will generate greater absolute frequency shifts for a given mass load. ${ }^{4}$ Therefore film bulk acoustic resonators are currently favoured over any other technology due to their much higher resonant frequency (hence greater sensitivity).[4, 5] FBAR has similar structure to QCM, but instead of quartz, a deposited piezoelectric ( $\mathrm{ZnO}$ or $\mathrm{AlN}$ ) thin film with a thickness typically around 1-2 $\mu \mathrm{m}$ is sandwiched by top and bottom electrodes. Due to the thinner piezoelectric layer, FBAR provides much higher resonant frequency. In comparison to QCM, SPR (Surface Plasmon Resonance) and other label-free biosensors such as spectroscopy and interferometry, FBAR requires less sample volume and can be integrated into CMOS-based high throughput arrays.[3, 6]

Since the first report of the use of FBARs as gravimetric biosensors for label-free detection of DNA and proteins by Gabl et al.[7], FBARs have been used as mass sensors for many biological applications. For example, Lee et al.[4] reported the use of AIN FBAR sensors for the detection of tumour marker carcinoembryonic antigens. Zhang et al.[8] demonstrated that FBARs can detect single base pair mismatch of the DNA hybridization. Auer et al.[9] recently reported that FBARs have the capability to detect $1 \mathrm{nM}$ oligonucleotide dissolved in $1 \%$ serum. In combination with a competitive antibody-antigen association process, it has been demonstrated that FBARs have the capability of detecting small drug molecules such as cocaine and heroin.[10] Recently, shear mode FBARs have been developed for the real-time monitoring of biomolecular binding under liquid environment. Weber et al.[11] pioneered the first real-time measurements of antibody-antigen binding in liquid using shear mode FBAR biosensors. Xu et al.[12, 13] have used FBAR sensors for the detection of aptamer-thrombin binding pair and for the real-time in situ monitoring of the competitive adsorption/exchange of proteins. Nirschl et al.[2, 6] also used FBAR sensors for the real-time detection of DNA hybridisation, antibody binding and recrystallisation of bacterial S layers. More recently, Chen et al. used the acetylcholinesterase-coated FBARs to detect the organophosphorus pesticides[14] and monoclonal anti-AFP antibody coated FABR for the detection of the cancer marker alpha-fetoprotein (AFP)[15]. All these applications are based on the shift of resonant frequency in response to the deposition or binding of biomolecules onto the biosensor electrode surfaces[16].

Prostate cancer is a major cancerous disease in the male population and accounts for about $10 \%$ of the deaths from cancers;[17-19] hence its early detection can save millions of lives. Monitoring the human Prostate-Specific Antigen (hPSA) level in serum is by far the most commonly used approach[19]. In the work reported here, FBARs have been fabricated as biosensors for the detection of antibody adsorption on a gold electrode surface and subsequent antigen binding using hPSA and anti-hPSA as model system. Previous research has reported the correlation of FBAR 
frequency shift to QCM and SPR results.[2, 6, 11] However, there is no sufficient study to correlate FBAR frequency shift to the real mass change of the biomolecules on the electrode surface.[5] Therefore, ellipsometry was employed in this work for the calibration of FBAR frequency changes into actual masses on the surface. The antibody adsorption behaviour on gold surface was firstly explored by ellipsometry to find the optimal conditions followed by parallel FBAR measurements. The high consistency of the data from two different techniques (optical and acoustical) indicates that FBARs are excellent candidate biosensors for biomedical applications such as cancer diagnostics.

\section{Experimental Section}

Materials. Phosphate buffers were made from $\mathrm{Na}_{2} \mathrm{HPO}_{4}$ and $\mathrm{NaH}_{2} \mathrm{PO}_{4}$ (Sigma, UK) with total ionic strength fixed at $20 \mathrm{mM}$. Mouse monoclonal anti-human Prostate-Specific Antigen (AntihPSA) and native human Prostate-Specific Antigen (hPSA) were purchased from AbD Serotec, Oxford, UK. Concentrations of the antibody and antigen were determined by UV at $A_{280}$ using the coefficients of 1.4 for the antibody and 1.84 for the antigen. Bovine serum albumin (BSA) was purchased from Sigma, UK. All samples were used as supplied. Au coated (100)-oriented Silicon substrates were made as described below for FBAR fabrication.

Spectroscopic ellipsometry (SE). The principle of ellipsometry has been described and can be found in the previous work.[20, 21] Gold coated silicon wafers (12 $\mathrm{mm} \times 12 \mathrm{~mm}$ ) were used in the ellipsometric cell (which accommodated $1 \mathrm{ml}$ sample solution) fitted with a pair of fused quartz windows[22] specially designed for solid/liquid interfacial measurements. The incoming and exiting beams were at $70^{\circ}$ to the surface normal and the windows were aligned to be perpendicular to the two beams. The substrates were cleaned and the thickness of the gold layer was determined before each measurement. Experiments of interfacial adsorption of the antibody, BSA blocking and antigen binding were carried out by using Jobin-Yvon UVISEL spectroscopic ellipsometer with a wavelength range between 300 and $600 \mathrm{~nm}$. Resulting data were analysed using the software DeltaPsi II developed by Jobin-Yvon Ltd. The surface adsorbed amount $\Gamma\left(\mathrm{mg} / \mathrm{m}^{2}\right)$ of the sample was calculated from $n_{f}$ and $\tau_{f}$ (in $\AA$ ) through Eq. 1[23, 24]

$$
\Gamma=\frac{\tau_{f}\left(n_{f}-n_{0}\right)}{(d n / d c)}
$$

where $n_{f}$ and $n_{0}$ are the refractive indexes of the film and buffer, $\tau_{f}$ is the thickness of the film and $d n / d c$ represents the change of refractive index against solution concentration and a value of 0.18 $\mathrm{ml} / \mathrm{g}$ was used in this work[20]. 
For a typical ellipsometric experiment, Anti-hPSA solution (5, 10 and $20 \mathrm{mg} / \mathrm{L}$ ) was first added into the ellipsometric cell with a gold coated wafer on the bottom. The adsorption of the antibody was allowed for different time scales from 5 min to $1 \mathrm{~h}$ and was stopped by replacing the antibody solution with buffer to wash off any loosely adsorbed molecules and to achieve different amount of antibody at the Au/buffer interface. BSA (50 mg/L) was then applied to the gold surface to block the non-specific adsorption for 25 min before buffer wash. Finally the antigen $(5 \mathrm{mg} / \mathrm{L}$ hPSA solution) was introduced to bind onto the surface immobilized antibodies. The values of antigen bind to the surface immobilized antibody were taken at 15 min binding. Each experiment (except the data in Figure 4) was repeated three times on the $5 \mathrm{~nm}$ gold coated wafers. Data in Figure 4 are individual data point obtained from both $5 \mathrm{~nm}$ and $50 \mathrm{~nm}$ gold coated surfaces.

FBAR fabrication. FBAR device fabrication is described elsewhere[25, 26]. Briefly, devices were fabricated on 4-in double-side-polished silicon substrates coated with a thin layer of thermally grown $\mathrm{SiO}_{2}$ (PI-KEM Ltd., Staffordshire, UK). The bottom electrodes (4/50 nm Cr/Au) were patterned through a standard photolithography process and thermally evaporated on top of the $\mathrm{SiO}_{2}$ surface. A $2 \mu \mathrm{m} \mathrm{ZnO} \mathrm{thin} \mathrm{film} \mathrm{was} \mathrm{then} \mathrm{deposited} \mathrm{using} \mathrm{high} \mathrm{target} \mathrm{utilisation} \mathrm{sputtering} \mathrm{(HiTUS).}$ This technique yields high quality films with very low stress and defect density, and homogeneity better than 99\% over the 4-in diameter area[27, 28]. The ZnO film was selectively wet etched in a 2\% glacial acetic acid and phosphoric acid solution to form a via for electrical connection to the bottom electrode. The top electrode, which was patterned with a second lift-off photolithography process, was evaporated with the same materials and thicknesses as the bottom electrode. Finally, the $\mathrm{SiO}_{2}$ on the back of the wafer was patterned and removed with $\mathrm{CF}_{4}$ plasma to expose the $\mathrm{Si}$, which was subsequently removed with a deep reactive ion etching (DRIE) process to release a freestanding membrane. The top $\mathrm{SiO}_{2}$ suspension layer serves as a barrier, preventing the $\mathrm{ZnO}$ film from being etched away with the DRIE process. This process provided a high fabrication yield (>95\%). The devices were then wire bonded onto $50 \Omega$ transmission line PCBs using Al wires. A schematic cross-sectional view of the FBAR device is shown in Figure 1A.

\section{Figure 1}

FBAR measurements. The bare FBAR sensors were ozoned for at least 40 min followed by UHQ (Ultra High Quality) water wash and $\mathrm{N}_{2}$ dry to clean the top electrodes surface. A schematic diagram of the experimental setup is shown in Figure 1B. Freshly cleaned sensors on $50 \Omega$ line PCB boards were then mounted inside the isolation box situated on an anti-vibration table and connected to an Agilent E5062A network analyzer (through S $_{21}$ channels) which was linked to a controller PC using the GPIB channel. The environmental temperature, which is known to affect the device 
response[28-31], was continuously controlled with a Haake bath (K20). The isolation box was then purged by dry nitrogen to remove humidity before each measurement step. Software written with LabVIEW® was used to continuously monitor and record the resonance spectrum of the FBAR. The frequency sweeps were performed with an excitation bandwidth of $1 \mathrm{MHz}$ around $f_{r}$ and a 1601-point resolution. The resonant frequency of the clean bare FBAR was recorded as base frequency. For a typical antibody adsorption experiment, a drop of $10 \mu \mathrm{l}$ antibody solution at different concentrations (5-50 mg/L) was introduced onto the clean electrode surfaces and allowed for adsorption to achieve different surface adsorbed amounts by controlling the adsorption time (2 min or $15 \mathrm{~min}$ ). Measurements were then carried out after buffer wash, gentle nitrogen drying of the sample and nitrogen purge of the isolation box. For the antigen binding experiment, using the similar procedure as describe for antibody adsorption, frequency changes were monitored after 15 min antibody adsorption, 25 min BSA blocking and 15 min antigen binding. A control experiment of BSA adsorption on electrode surface (30 min) and antigen adsorption (15 min) on top of BSA was also carried out to investigate the non-specific binding of the antigen. At least three repeat runs were carried out for each step and the results were averaged. The standard deviation of three measurements is typically less than $5 \mathrm{kHz}$.

\section{Results and Discussion}

\subsection{Antibody dynamic adsorption on gold surface and antigen binding}

\section{Figure 2}

Protein adsorption is a dynamic process and the amount of protein adsorbed on surface is concentration and time dependent.[32, 33] While the packing density of the protein and its orientation on the substrate surface are crucial to biomarker detection[16, 32, 34], finding the right protein immobilization conditions is important for improving the biosensor performances. Spectroscopic ellipsometry (SE) is a convenient method for monitoring the dynamic interfacial adsorption of biomolecules.[20, 32, 34-37] Therefore, it has been used in this study for the detection of antibody adsorption and subsequent antigen binding to screen the optimal conditions for the subsequent biosensor measurements.

Figure 2 shows the dynamic adsorption of Anti-hPSA antibody at the Au/buffer interface and the subsequent BSA blocking and hPSA antigen binding. At all concentrations studied, the surfaceadsorbed amount of the antibody steadily increases with time and then tends to a plateau. The higher concentration tends to the plateau faster and achieves higher surface adsorbed amount. However, even after being equilibrated for $1 \mathrm{~h}$, the adsorbed amount has not reached the 
equilibrated plateau yet, indicating the slower adsorption process of the antibody on gold surface when compared with that on silicon dioxide surface.[32] In our previous study, it was found that antibody with concentrations at 10 and $20 \mathrm{mg} / \mathrm{L}$ almost reached the adsorption plateau on silicon dioxide surface at $1 \mathrm{~h}$. At the concentrations below $10 \mathrm{mg} / \mathrm{L}$, the surface-adsorbed amounts of antibody at $1 \mathrm{~h}$ on gold surface $\left(2 \mathrm{mg} / \mathrm{m}^{2}\right.$ at $5 \mathrm{mg} / \mathrm{L}$ and $2.6 \mathrm{mg} / \mathrm{m}^{2}$ at $\left.10 \mathrm{mg} / \mathrm{L}\right)$ are much greater than that on silicon dioxide surface ( 1 and $2 \mathrm{mg} / \mathrm{m}^{2}$, respectively), while at the concentration of 20 $\mathrm{mg} / \mathrm{L}$ the surface adsorbed amounts (at $1 \mathrm{~h}$ ) on the two surfaces are similar $\left(3.1 \mathrm{mg} / \mathrm{m}^{2}\right)$, indicating the different dynamic processes as a result of different surface chemical nature.

The adsorption was then stopped at different times by replacing the antibody solution with buffer to wash off any loosely adsorbed molecules and to achieve different amount of antibody at the Au/buffer interface. Evidence from Figure 2 suggests that desorption is negligible, indicating the strong binding between gold surface and antibody molecules. Similarly to many immunoassays, higher concentration BSA (50 mg/L) was then used to block any non-specific adsorption, i.e., the possible binding that was not caused by the specific binding of epitopes. For example, at low antibody coverage (below $2 \mathrm{mg} / \mathrm{m}^{2}$ ), there are some spaces that have not been covered by antibody molecules.[32] Smaller molecules such as BSA (68 kDa) can fill in the gaps and block any electrostatic and hydrophobic interactions. In Figure 2, a distinct increase of BSA adsorption can be observed when the adsorbed amount of antibody is below $2 \mathrm{mg} / \mathrm{m}^{2}$. This observation is consistent with that at the $\mathrm{SiO}_{2}$ /buffer interface, where the antibody molecules predominantly lied flat on the surface[32]. As the antibodies have the approximate dimensions of $142 \AA \times 85 \AA \times 38 \AA$, a single antibody molecule occupies an area of $12100 \AA^{2}$ and a full monolayer coverage with flat-on orientation on the surface results in a surface-adsorbed amount of $2 \mathrm{mg} / \mathrm{m}^{2}$.[32] Above this amount no noticeable BSA adsorption was observed, indicating the full occupation of the surface by antibody molecules. This interfacial structural feature has been confirmed by both neutron reflection experiments in our previous study[32] and the results of monolayer footprints of antibody on glass beads and silica microparticles[38]. The molecular adsorbed surface was then rinsed again for 15 min by buffer after 25 min BSA blocking before the final antigen binding. Increase in the adsorbed amount was detected after applying the antigen solution, indicating the occurrence of antigen binding. Figure 2 also shows that the antigen binding amount was relatively higher when the amount of antibody on gold surface was around $1( \pm 0.5) \mathrm{mg} / \mathrm{m}^{2}$.

\section{Figure 3}

To demonstrate the relationship between the antibody surface-adsorbed amount and solution concentrations, Figure 3 shows the surface-adsorbed amount of antibody (all at $1 \mathrm{~h}$ ) at different 
concentrations. It depicts a dramatic increase of the antibody surface adsorption with its bulk concentration below $20 \mathrm{mg} / \mathrm{L}$, above which the extent of increase of the adsorbed amount slowed down and tended to an adsorption plateau. This observation is consistent with the recent work by Chen et al.[39]. They found that the SPR angle increased dramatically from the anti-hCG (human chorionic gonadotrophin) antibody adsorption within the concentration range between 2 to $20 \mathrm{mg} / \mathrm{L}$. Above this concentration the rate of the SPR angle rise was much slower, indicating the attainment of antibody adsorption plateau. This feature of adsorption plateau has also been observed from antibody adsorption at the $\mathrm{SiO}_{2} /$ buffer interface[32, 40, 41], although the slopes of the adsorption curves below $10 \mathrm{mg} / \mathrm{L}$ can differ due to the different surface effects.

Figure 3 also displays the antigen binding amount and binding ratio after $1 \mathrm{~h}$ adsorption of antibody at different concentrations. The antigen binding ratio was calculated based on the molar ratio between the antigen and the binding sites on antibody (e.g. binding ratio = mole of antigens / (mole of antibody $\times 2)$ ). It is clear that both the amount and binding ratio decrease dramatically with increasing antibody concentration. After $5 \mathrm{mg} / \mathrm{L}$ or when the surface-adsorbed amount of antibody is above $2 \mathrm{mg} / \mathrm{m}^{2}$, the binding amount and binding ratio level off. These observations indicate the occurrence of higher antigen binding amount and ratio in lower antibody surface coverage, typically below $2 \mathrm{mg} / \mathrm{m}^{2}$, a common feature of hPSA and hCG binding systems.[32, 41, 42] With a lower amount of antibody molecules adsorbed at the interface, the accessibility is greater, resulting in better binding efficiency. Increasing the antibody amount at the interface causes higher steric hindrance, producing lower binding efficiency. Because the surface packing density of antibody is crucial to the antigen binding, we have undertaken a detailed study to find the optimal condition for antigen binding.

\subsection{Optimization of antigen binding to the immobilized antibodies on gold surfaces}

\section{Figure 4}

Figure 4 shows the surface-adsorbed amount of antibody, the amount of antigen bound, and the ratio of the two. Extensive work was devoted to improving the antigen binding amount and the ratio by adjusting the parking density of antibody at the Au/buffer interface. As seen from Figure 4, the amount of antigen bound to the immobilized antibody increased with the increasing amount of antibody initially, then reached the maximum, before it became declined and then levelled off. The maximum occurred at the antibody packing density around $1 \mathrm{mg} / \mathrm{m}^{2}$. However, the binding ratio showed a drastic decline with increasing amount of the antibody, indicating the undermining effect of surface crowding. Similar feature was also observed at the $\mathrm{SiO}_{2} /$ buffer interface with the maximum occurring at the antibody packing density around $1.2 \mathrm{mg} / \mathrm{m}^{2}$.[32] Previous neutron 
reflection and AFM studies have revealed that under these conditions antibody molecules adsorbed at the silicon oxide/water interface predominantly adopt flat-on conformation (with the 2 Fabs and Fc all in contact with the surface).[32, 40, 41, 43, 44] As mentioned above, a full monolayer coverage of the antibodies is equivalent to a surface-adsorbed amount of approximately $2 \mathrm{mg} / \mathrm{m}^{2}$, below which the binding sites have very good accessibility. Therefore, the binding ratio was high. As it can be seen in Figure 4, when the amount of antibody is less than $0.7 \mathrm{mg} / \mathrm{m}^{2}$ the binding ratio is over 0.9. With increasing the amount of antibody at the interface, the amount of antigen bond to the immobilized antibody increased due to the increasing binding sites. However, the binding ratio starts to decline due to the gradual increase of the steric hindrance. There is a balance between the increase of binding sites and the reduction of accessibility. Therefore, a maximum occurred at 1 $\mathrm{mg} / \mathrm{m}^{2}$ which was equivalent to $50 \%$ of the monolayer coverage of the surface. Above this value, both the binding amount and the binding ratio reduced, indicating that the steric hindrance was becoming dominant. Above $2 \mathrm{mg} / \mathrm{m}^{2}$, the surface is fully covered by the antibody and multilayer adsorption starts to occur. Many of the binding sites buried inside the layer are unavailable for antigen binding. Therefore, the binding efficiency becomes very low and the amount of the bound antigen tends to be irrelevant to the amount of antibody adsorbed, a general feature observed from several other antibody-antigen binding systems[32, 41, 42]. Similar findings were observed by Yuan et al.[45] using SPR when they tried to improve the immobilization capacity of anti-rabbit IgG and antigen binding using chitosan/alginate layer-by-layer self-assembled multilayer films. Le et al.[46] also confirmed, by using total internal reflection ellipsometry, that an increase in surfaceadsorbed anti-R-hCG resulted in the reduction of hCG binding efficiency.

It is useful to note that the data obtained from the $5 \mathrm{~nm}$ gold layer highly agree with the data obtained from $50 \mathrm{~nm}$ gold layer. The latter has been used as the top electrodes of FBAR sensors. Therefore, the data from ellipsometry can be used for the calibration of the FBAR frequency shifts.

\subsection{FBAR as gravimetric biosensors}

\subsubsection{FBAR characterization and response to antibody absorption}

\section{Figure 5}

Electrical characterization of the FBAR devices was carried out using an Agilent E5062A network analyser. A typical response at around $1.56 \mathrm{GHz}$ with an insertion loss of around $-30 \mathrm{~dB}$ is shown in Figure $5 \mathrm{~A}$ as an example. The sharp resonance of the $\mathrm{ZnO}$ indicates a very high quality factor $(Q)$ which was determined by measuring the transmission signal $\left(\mathrm{S}_{21}\right)$ and $3 \mathrm{~dB}$ bandwidth [47-50] using Eq. (2) 


$$
Q_{-3 d B}=\frac{f_{0}}{\Delta f_{-3 d B}}
$$

where $f_{0}$ is the resonant frequency and $f_{\text {-3dB }}$ is the bandwidth at $3 \mathrm{~dB}$, both extracted from the transmission signal spectrum. The typical $3 \mathrm{~dB} Q$ of the FBAR fabricated was found to be around 860. Continuous monitoring of the resonant frequency of a time scale of 800 s demonstrates that the FBARs have very low frequency indeterminacy, typically around $3 \mathrm{kHz}$ as shown in Figure 5B.

After characterization of the FBARs, protein solutions were applied onto the active area of the devices and incubated for different durations followed by buffer rinsing and nitrogen drying. At least three measurements were carried out for each experimental step and the data were averaged with a standard deviation of less than $5 \mathrm{kHz}$. As an example, Figure 5C shows experimentally measured curves of frequency sweeps with 1601 data points in each curve. The lowest point of each curve was recorded for the calculation of the frequency change. The right curve is the resonant tip of a clean bare FBAR while the left curve is the resonant tip after antibody adsorption. The adsorption of $50 \mathrm{mg} / \mathrm{L}$ antibody on the FBAR surface for 15 min resulted in the tip frequency shiftdown of around $600 \mathrm{kHz}$ and the reduction of insertion loss of $1 \mathrm{~dB}$ due to the protein deposition onto the gold electrode surface. Both the high $Q$ factor and low frequency indeterminacy enable the accuracy of the measurements.

\subsubsection{Calibration of the FBAR frequency shift to the adsorbed antibody amount by ellipsometry}

\section{Figure 6}

FBARs have been widely used as gravimetric biosensors[2, 12, 13, 51]. We have also demonstrated FBAR frequency responses to the mass loading of BSA at different concentrations[25, 52, 53] and to the capture of DEET (N,N-Diethyl-meta-toluamide) molecules by the pre-immobilized odorant binding protein originated from Aedes aegypti mosquitoes[16]. To correlate the FBAR frequency shift to the amount of antibody adsorbed on the electrode surface in this work, ellipsometric measurements were carried out in parallel to the FBAR measurements and the data are shown in Figure 6. Measurements were carried out using antibody concentrations ranged from 5 to $50 \mathrm{mg} / \mathrm{L}$ for the adsorption of 2 and 15 min to achieve different surface adsorbed amount of antibodies. The combination of different adsorption times and concentrations ensured the data covering a wide range of surface adsorbed amount from 0.3 to $3 \mathrm{mg} / \mathrm{m}^{2}$ thereby ensure a nice calibration of FBAR frequency change against surface adsorbed amount. It was found that within the solution concentration range and adsorption durations studied, both the frequency shift of FBAR and the 
surface adsorbed amount obtained from ellipsometry were linear to the antibody concentrations and the FBAR data was in remarkable agreement with the ellipsometry data. It was found from Figure 6b that a frequency shift of $200 \mathrm{kHz}$ is equivalent to $1 \mathrm{mg} / \mathrm{m}^{2}$ which gives a mass sensitivity of 2 $\mathrm{kHz} \mathrm{cm}^{2} / \mathrm{ng}$, equivalent to the findings by Nirschl et al.[2] who have also achieved a mass sensitivity of $\sim 2 \mathrm{kHz} \mathrm{cm} / 2$ ng using shear mode FBARs. As the frequency indeterminacy is $3 \mathrm{kHz}$, this gives a resolution of $0.015 \mathrm{mg} / \mathrm{m}^{2}\left(1.5 \mathrm{ng} / \mathrm{cm}^{2}\right)$ comparable to the mass resolutions (1.35 and $\left.1.78 \mathrm{ng} / \mathrm{cm}^{2}\right)$ found by Xu et al.[12, 13], $\left(2.3 \mathrm{ng} / \mathrm{cm}^{2}\right)$ Weber et al.[11] and (1 ng/cm $\left.{ }^{2}\right)$ Nirschl et al.[2]. This mass sensitivity is also consistent with the value in Wingqvist's review[1] (0.3 -7.5 $\mathrm{ng} / \mathrm{cm}^{2}$ ). This result demonstrates that the FBARs have the capability to be employed as high precision biosensors.

\subsection{Label-free detection of antigen binding using FBAR sensors}

\section{Figure 7}

The FBARs were subsequently used for the detection of hPSA using surface immobilized monoclonal antibodies on the top electrode surface. Figure 7 shows the resonant frequency shifts after antibody adsorption, BSA blocking and antigen binding. As has been shown in Figure 4, the best condition for the high amount of antigen binding is at the surface immobilized antibody around $1 \mathrm{mg} / \mathrm{m}^{2}$. Based on the ellipsometry calibration data from Figure 6, this can be achieved by the adsorption either from $5 \mathrm{mg} / \mathrm{L}$ antibody solution for $15 \mathrm{~min}$ or $20 \mathrm{mg} / \mathrm{L}$ solution for $2 \mathrm{~min}$. As shown in Figure 7, the adsorption of $5 \mathrm{mg} / \mathrm{L}$ solution for $15 \mathrm{~min}$ resulted in a frequency shift down of around $230 \mathrm{kHz}$, equivalent to a surface adsorbed amount of antibody around $1.1 \mathrm{mg} / \mathrm{m}^{2}$. It can be seen from Figure 2 that it took about 20 min for BSA to reach the equilibrium blocking. Therefore, 25 min (same duration as ellipsometry measurements shown in Figure 2) was used for BSA blocking which resulted in a further frequency reduction of around $70 \mathrm{kHz}$. Meanwhile, parallel to ellipsometric measurements, 15 min was allowed for the antigen to bind to the antibody on the electrode surface before buffer wash and nitrogen dry. The antigen binding resulted in another $46 \mathrm{kHz}$ shift, equivalent to an amount of antigen of $0.23 \mathrm{mg} / \mathrm{m}^{2}$. These values are highly consistent with the results shown in Figure 4 by ellipsometry. The optimal antigen binding amount are also consistent with the data at the silica/buffer interface revealed by ellipsometry, neutron reflection and Dual Polarization Interferometry (DPI). It was found by the three techniques that at the optimal condition, the antigen binding amount was $0.22 \pm 0.05 \mathrm{mg} / \mathrm{m}^{2}$.[32, 34] A control experiment of non-specific adsorption of hPSA antigen on top of BSA was also carried out and the data are shown in Figure 7. Adsorption of BSA $(50 \mathrm{mg} / \mathrm{L})$ on the electrode surface for $30 \mathrm{~min}$ resulted in a frequency shift down of $71 \mathrm{kHz}$ which is highly consistent with the result in blocking 
step. Further adsorption of hPSA antigen (5mg/L for $15 \mathrm{~min}$ ) on top of BSA only resulted in $2 \mathrm{kHz}$ of frequency shit indicating the negligible non-specific antigen adsorption on BSA. These results demonstrate that FBAR can precisely detect antibody adsorption and antigen binding.

\section{Conclusion}

Label-free detection of cancer biomarkers using low cost and disposable biosensors is promising in cancer diagnostics and many other clinical applications. In this paper, the adsorption and antigen binding behaviour of monoclonal antibody on gold surface has been optimized using ellipsometry. $\mathrm{ZnO}$ thin film bulk acoustic resonators (FBARs) with a mass sensitivity of $0.015 \mathrm{mg} / \mathrm{m}^{2}(1.5$ $\mathrm{ng} / \mathrm{cm}^{2}$ ) have been demonstrated for their potential use as an immunosensor. Our FBARs were designed to exhibit high resonant frequencies ( 1.5 GHz) and high $Q$ factors (typically around 800). The frequency indeterminacy was found to be less than $3 \mathrm{kHz}$ and the standard deviation of three measurements is typically less than $5 \mathrm{kHz}$. Compared to a number of existing literatures that have simply reported FBAR responses to several biomarkers without validating their results to real mass changes, we have, for the first time, done so and demonstrated the validity of this method for biomarker quantification through parallel ellipsometric measurements. It was found that both the frequency shift of FBAR and the surface adsorbed amount obtained from ellipsometry are linear to the antibody concentration within the timescale and concentrations studied. And the correlation factor was found to be $200 \mathrm{kHz} \mathrm{m} / \mathrm{mg}$ ( $2 \mathrm{kHz} \mathrm{cm} / \mathrm{ng}$ ). Subsequent antigen detection experiment demonstrated that FBARs can precisely detect the antigen binding to the antibody pre-immobilized on the FBAR gold electrode surface. Therefore, the current study suggests that FBARs have great potential as label-free and disposable biosensors.

\section{Acknowledgment}

The authors wish to thank EPSRC for financial support under grants EP/F062966/1, EP/F063865/1 and EP/F06294X/1. XZ also wish to thank The Royal Society for financial support through grant RG120061. LGG acknowledges additional financial support from the National Natural Science Foundation of China (NSFC) through grant number 61150110485.

\section{References}

[1] G. Wingqvist, AlN-based sputter-deposited shear mode thin film bulk acoustic resonator (FBAR) for biosensor applications - A review, Surface \& Coatings Technology, 205(2010) 1279-86.

[2] M. Nirschl, A. Blüherc, C. Erler, B. Katzschnerc, I. Vikholm-Lundind, S. Auerd, et al., Film bulk acoustic resonators for DNA and protein detection and investigation of in vitro bacterial Slayer formation, Sensors and Actuators A: Physical, 156(2009) 180-4.

[3] K. Tukkiniemi, A. Rantala, M. Nirschl, D. Pitzer, T. Huber, M. Schreiter, Fully integrated FBAR sensor matrix for mass detection, Procedia Chemistry, 1(2009) 1051-4. 
[4] T.Y. Lee, J.T. Song, Detection of carcinoembryonic antigen using AlN FBAR, Thin Solid Films, 518(2010) 6630-3.

[5] G. Wingqvist, V. Yantchev, I. Katardjiev, Mass sensitivity of multilayer thin film resonant BAWsensors, Sensors and Actuators A: Physical, 148(2008) 88-95.

[6] M. Nirschl, A. Rantala, K. Tukkiniemi, S. Auer, A.-C. Hellgren, D. Pitzer, et al., CMOSIntegrated Film Bulk Acoustic Resonators for Label-Free Biosensing, Sensors, 10(2010) 4180-93.

[7] R. Gabl, H.-D. Feucht, H. Zeininger, G. Eckstein, M. Schreiter, R. Primig, et al., First results on label-free detection of DNA and protein molecules using a novel integrated sensor technology based on gravimetric detection principles, Biosensors and Bioelectronics, 19(2004) 615-20.

[8] H. Zhang, M.S. Marma, S.K. Bahl, E.S. Kim, C.E. McKenna, Sequence Specific Label-Free DNA Sensing Using Film-Bulk-Acoustic-Resonators, IEEE Sensors J, 7(2007) 1587-8.

[9] S. Auer, M. Nirschl, M. Schreiter, I. Vikholm-Lundin, Detection of DNA hybridisation in a diluted serum matrix by surface plasmon resonance and film bulk acoustic resonators, Anal Bioanal Chem, 400(2011) 1387-96.

[10] G. Wingqvist, J. Bjurströma, A.-C. Hellgren, I. Katardjiev, Immunosensor utilizing a shear mode thin film bulk acoustic sensor, Sensors and Actuators B: Chemical, 127(2007) 248-52.

[11] J. Weber, W.M. Albers, J. Tuppurainen, M. Link, R. Gabl, W. Wersing, et al., Shear mode FBARs as highly sensitive liquid biosensors, Sensors and Actuators A: Physical, 128(2006) 84-8.

[12] W. Xu, S. Choi, J. Chae, A contour-mode film bulk acoustic resonator of high quality factor in a liquid environment for biosensing applications, Applied Physics Letters, 96(2010) 053703.

[13] W. Xu, X. Zhang, S. Choi, J. Chae, A High-Quality-Factor Film Bulk Acoustic Resonator in Liquid for Biosensing Applications, Journal of Microelectromechanical Systems, 20(2011) 213-20.

[14] D. Chen, J. Wang, Y. Xu, D. Li, L. Zhang, Z. Li, Highly sensitive detection of organophosphorus pesticides by acetylcholinesterase-coated thin film bulk acoustic resonator massloading sensor, Biosensors \& bioelectronics, 41(2013) 163-7.

[15] D. Chen, J.J. Wang, D.H. Li, Z.X. Li, Film bulk acoustic resonator based biosensor for detection of cancer serological marker, Electronics Letters 47(2011) 1169-70.

[16] X. Zhao, G.M. Ashley, L. Garcia-Gancedo, H. Jin, J. Luo, A.J. Flewitt, et al., Protein Functionalized ZnO Thin film bulk acoustic resonator as an Odorant Biosensor, Sensors and Actuators B: Chemical, 163(2012) 242-6.

[17] J.M. Choi, J.Y. An, B.W. Kim, Effect of Biolinker on the Detection of Prostate Specific Antigen in an Interferometry, Biotechnology and Bioprocess Engineering, 14(2009) 6-12.

[18] C. Cao, J.P. Kim, B.W. Kim, H. Chae, H.C. Yoon, S.S. Yang, et al., A strategy for sensitivity and specificity enhancements in prostate specific antigen-alpha1-antichymotrypsin detection based on surface plasmon resonance, Biosensors and Bioelectronics, 21(2006) 2106-13.

[19] D.A. Healy, C.J. Hayes, P. Leonard, L. McKenna, R. O'Kennedy, Biosensor developments: application to prostate-specific antigen detection, TRENDS in Biotechnology, 25(2007) 125-31.

[20] X. Zhao, F. Pan, P. Coffey, J.R. Lu, Cationic Copolymer-Mediated DNA Immobilization: Interfacial Structure and Composition As Determined by Ellipsometry, Dual Polarization Interferometry, and Neutron Reflection, Langmuir, 24(2008) 13556-64.

[21] F. Pan, X. Zhao, S. Perumal, T.A. Waigh, J.R. Lu, J.R.P. Webster, Interfacial Dynamic Adsorption and Structure of Molecular Layers of Peptide Surfactants, Langmuir, 26(2010) 5690-6.

[22] Y. Tang, J.R. Lu, A.L. Lewis, T.A. Vick, P.W. Stratford, Swelling of zwitterionic polymer films characterized by spectroscopic ellipsometry, Macromolecules, 34(2001) 8768-76.

[23] J.A. De-Feijter, J. Benjamins, F.A. Veer, Ellipsometry as a tool to study the adsorption behavior of synthetic and biopolymers at the air-water interface, Biopolymers, 17(1978) 1759-72.

[24] M. Martin, Ellipsometry studies of protein layers adsorbed at hydrophobic surfaces, J Colloid Interface Sci, 166(1994) 333-42.

[25] L. García-Gancedo, G.M. Ashley, X.B. Zhao, J. Pedrós, A.J. Flewitt, W.I. Milne, et al., Deposition and characterisation of ultralow-stress $\mathrm{ZnO}$ thin films for application in FBAR-based gravimetric biosensors International Journal of Nanomanufacturing, 7(2011) 371-82. 
[26] L. García-Gancedo, J. Pedrós, X.B. Zhao, G.M. Ashley, A.J. Flewitt, W.I. Milne, et al., Dualmode thin film bulk acoustic wave resonators for parallel sensing of temperature and mass loading, Biosensors and Bioelectronics, 38(2012) 369-74.

[27] L. García-Gancedo, J. Pedrós, Z. Zhu, A.J. Flewitt, W.I. Milne, J.K. Luo, et al., Roomtemperature remote-plasma sputtering of c-axis oriented zinc oxide thin films, J Appl Phys, 112(2012) 014907.

[28] L. Garcia-Gancedo, J. Pedrós, A.J. Flewitt, W.I. Milne, G.M. Ashley, J.K. Luo, et al., Ultrafast sputtered $\mathrm{ZnO}$ films with high kT for acoustic wave device applications, IEEE Ultrasonics Symposium, (2010) 1064-7.

[29] S. Rai, Y. Su, W. Pang, R. Ruby, B. Otis, A digitally compensated 1.5 GHz CMOS/FBAR frequency reference, IEEE Ultras Ferro Freq Contr, 57(2010) 552-61.

[30] J. Bjurstrom, G. Wingqvist, V. Yantchev, I. Katardjiev, Temperature compensation of liquid FBAR sensors, J Micromech Microeng, 17(2007) 651-8.

[31] L. García-Gancedo, J. Pedrós, X. Zhao, G. Ashley, A. Flewitt, W. Milne, et al., Dual-mode thin film bulk acoustic wave resonators for parallel sensing of temperature and mass loading, Biosensors \& bioelectronics, 38(2012) 369-74.

[32] X. Zhao, F. Pan, B. Cowsill, J.R. Lu, L. Garcia-Gancedo, A.J. Flewitt, et al., Interfacial Immobilization of Monoclonal Antibody and Detection of Human Prostate-Specific Antigen, Langmuir, 27(2011) 7654-62.

[33] F. Pan, X. Zhao, T.A. waigh, J.R. Lu, F. Miano, Interfacial adsorption and denaturization of human milk and recombinant rice lactoferrin, Biointerphases, 3(2008) FB36-FB43.

[34] X. Zhao, F. Pan, L. Garcia-Gancedo, A.J. Flewitt, G.M. Ashley, J. Luo, et al., Interfacial Recognition of Human Prostate-Specific Antigen by Immobilized Monoclonal Antibody: Effects of Solution Conditions and Surface Chemistry, J R Soc Interface, 9(2012) 2457-67.

[35] X. Zhao, F. Pan, S. Perumal, H. Xu, J.R. Lu, J.R.P. Webster, Interfacial Assembly of Cationic Peptide Surfactants, Soft Matter, 5(2009) 1630-8.

[36] X. Zhao, Z. Zhang, F. Pan, Y. Ma, S.P. Armes, A.L. Lewis, et al., DNA immobilization using biocompatible diblock phosphorylcholine copolymers, Surf Interface Anal, 38(2006) 548-51.

[37] X. Zhao, Z. Zhang, F. Pan, Y. Ma, S.P. Armes, A.L. Lewis, et al., Solution pH-regulated interfacial adsorption of diblock phosphorylcholine copolymers, Langmuir, 21(2005) 9597-603.

[38] M. Hoehne, F. Samuel, A. Dong, C. Wurth, H.-C. Mahler, J.F. Carpenter, et al., Adsorption of Monoclonal Antibodies to Glass Microparticles, Journal of Pharmaceutical Sciences, 100(2011) 123-32.

[39] H. Chen, J. Huang, J. Lee, S. Hwang, K. Koh, Surface plasmon resonance spectroscopic characterization of antibody orientation and activity on the calixarene monolayer, Sensors and Actuators B, 147(2010) 548-33.

[40] H. Xu, X. Zhao, C. Grant, J.R. Lu, D.E. Williams, J. Penfold, Orientation of a Monoclonal Antibody Adsorbed at the Solid/Solution Interface: A Combined Study Using Atomic Force Microscopy and Neutron Reflectivity, Langmuir, 22(2006) 6313-20.

[41] H. Xu, J.R. Lu, D.E. Williams, Effect of Surface Packing Density of Interfacially Adsorbed Monoclonal Antibody on the Binding of Hormonal Antigen Human Chorionic Gonadotrophin, J Phys Chem B, 110(2006) 1907-14.

[42] X. Wang, Y. Wang, H. Xu, H. Shan, J.R. Lu, Dynamic adsorption of monoclonal antibody layers on hydrophilic silica surface: A combined study by spectroscopic ellipsometry and AFM, J Colloid Interface Sci, 323(2008) 18-25.

[43] H. Xu, X. Zhao, J.R. Lu, D.E. Williams, Relationship between the Structural Conformation of Monoclonal Antibody Layers and Antigen Binding Capacity, Biomacromolecules, 8(2007) 2422-8.

[44] X. Zhao, F. Pan, J.R. Lu, Interfacial assembly of proteins and peptides: recent examples studied by neutron reflection, J R Soc Interface, 6(2009) S659-S70.

[45] W. Yuan, H. Dong, C.M. Li, X. Cui, L. Yu, Z. Lu, et al., pH-Controlled Construction of Chitosan/Alginate Multilayer Film: Characterization and Application for Antibody Immobilization, Langmuir, 23(2007) 13046-52. 
[46] N.C.H. Le, V. Gubala, R.P. Gandhiraman, C. Coyle, S. Daniels, D.E. Williams, Total internal reflection ellipsometry as a label-free assessment method for optimization of the reactive surface of bioassay devices based on a functionalized cycloolefin polymer, Anal Bioanal Chem, 398(2010) 1927-36.

[47] K.M. Lakin, G.R. Kline, K.T. McCarron, High-Q microwave acoustic resonators and filters, IEEE Trans Microwave Theory Tech, 41(1993) 2139-46.

[48] A.D. Wathen, F. Munir, W.D. Hunt, A high-Q hybrid acoustic mode in thin film ZnO solidly mounted resonators, Applied Physics Letters, 95(2009) 123509.

[49] J.K. Coakley, J.D. Splett, M.D. Janezic, R.F. Kaiser, Estimation of Q-Factors and Resonant Frequencies, IEEE Transactions on Microwave Theory and Techniques, 51(2003) 862-8.

[50] J.D. LarsonIII, P.D.B. SM, S.W. SM, R.C. Ruby, Modified Butterworth-Van Dyke Circuit for FBAR Resonators and Automated Measurement System, Proc-IEEE Ultrason Symp, 861(2000) 863-8.

[51] L. Mai, N.-H. Do, T.-A. Hoang, V.-S. Tran, L.-P.B. Pham, T.-T.N. Thi, A feasibility study of ZnO-based FBAR devices for an ultra-mass-sensitive sensor application, Microwave and Optical Technology Letters 42(2004) 505-7.

[52] L. García-Gancedo, E. Iborra, M. Clement, J. Olivares, Z. Zhu, A.J. Flewitt, et al., Solidly mounted resonators with carbon nanotube electrodes for biosensing applications, Frequency Control and the European Frequency and Time Forum (FCS), 2011 Joint Conference of the IEEE International (2011) 1-5

[53] L. García-Gancedo, Z. Zhu, E. Iborra, M. Clement, J. Olivares, A.J. Flewitt, et al., AlN-based BAW resonators with CNT electrodes for gravimetric biosensing, Sensors and Actuators B: Chemical, 160(2011) 1386-93.

Vitae

Xiubo Zhao is a Lecturer in the Department of Chemical and Biological Engineering at the University of Sheffield. He received his Ph.D. degree in biological physics from the University of Manchester in Dec 2006 after which he worked as an EPSRC funded Postdoctoral Research Fellow within the University of Manchester. His research interests include biomarker immobilisation and detection for biosensoring, peptide self-assembly, non-viral gene and drug delivery, interfacial adsorption of biomolecules, biomaterials and surface biocompatibility. Dr Zhao is a member of the Institute of Physics, UK.

Fang Pan received her B.M. degree From YangZhou University in 2004 and her PhD degree in biological physics from the University of Manchester in 2009. She is currently a KTP research associate in the biological physics group at the University of Manchester. Her research interests include peptide self-assembly; non-viral gene delivery; biomolecular adsorption; biomaterials, biocompatible and thermoresponsive polymer for tissue engineering.

Gregory M. Ashley received his B.Sc. degree in microbiology from the University of Liverpool in 1996, then M.Sc. degree in Biosensors from the University of Manchester in 2000. He went on to obtain his Ph.D. in Biosensors in the University of Cranfield on RF MEMS based Biosensors. He is at present a Postdoctoral Research Fellow at the University of Bolton, working on FBAR based biosensors.

Luis García-Gancedo received a B.Sc. in physics from the University of Oviedo, Spain in 2003. He then joined the University of Brighton (UK) where he received a Ph.D. for his work on magnetostrictive ultrasonic transducers for SONAR applications. After completing his Ph.D., Luis 
worked as a Research Fellow at the University of Birmingham (UK), in a multidisciplinary project fabricating ultrasonic transducers and arrays for ultrahigh resolution real time biomedical imaging. He joined Cambridge University (UK) in January 2009 and is at present a Research Associate at the Electrical Engineering Division, working on the development of thin film bulk acoustic resonators (FBARs) based ultrahigh sensitive biosensor arrays for diagnostic applications. Since October 2010 Luis is also a Lecturer in Engineering at Newnham College, University of Cambridge.

Jack Luo received his Ph.D. from the University of Hokkaido, Japan in 1989. He worked in Cardiff University as a research fellow, in Newport Wafer Fab. Ltd., Philips Semiconductor Co. and Cavendish Kinetics Ltd. as an engineer, senior engineer and manager, and then in Cambridge University as a senior researcher from 2004. From January 2007, he became a Professor in MEMS at the Centre for Material Research and Innovation (CMRI), University of Bolton. His current research interests focus on microsystems and sensors for biotechnology and healthcare applications, and third generation thin film solar cells using novel low cost materials.

Andrew J. Flewitt received the B.Sc. degree in physics from the University of Birmingham, U.K. in 1994 and the Ph.D. degree in scanning tunnelling microscopy of amorphous silicon from the University of Cambridge in 1998. Following this, he was a Research Associate studying the lowtemperature growth of silicon-based materials in the Engineering Department, University of Cambridge. He was appointed to a Lectureship in the same Department in 2002. Since 2009, he has held the position of University Reader in Electronic Engineering. His research interests span a broad range of large area electronics and related fields, including thin film transistors and MEMS devices. Dr. Flewitt is a Chartered Physicist and a Member of the Institute of Physics and the Institution of Engineering and Technology.

Bill I. Milne FREng, FIET, FIMMM has been Head of the Electrical Engineering Division at Cambridge University since 1999 and Director of the Centre for Advanced Photonics and Electronics (CAPE) since 2004. He obtained his BSc from St Andrews University in Scotland in 1970 and then went on to read for a Ph.D. in Electronic Materials at Imperial College London. He was awarded his Ph.D. and DIC in 1973 and in 2003, a D. Eng (Honoris Causa) from University of Waterloo, Canada. He was elected as Fellow of the Royal Academy of Engineering in 2006 and was awarded the JJ Thomson medal from the IET in 2008. He is a Guest Professor at HuangZhou University in Wuhan, China and a Distinguished Visiting Professor at SEU in Nanjing, China and at NUS, Singapore in 2010. He is also a Distinguished Visiting Scholar at KyungHee University, Seoul. He has published/presented $~ 750$ papers, of which 150 were invited.

Jian R. Lu is professor of biological physics and head of the Biological Physics Group at the University of Manchester. He obtained his Ph.D. degree from Hull University in 1991 in surface chemistry and went on a post-doctoral fellowship in Physical and Theoretical Chemistry Laboratory at Oxford University. From 1995 to 2000 he worked as Lecturer and Reader at Surrey University before taking the chair at Manchester in 2001. He has developed an international reputation in the use of neutron reflection and related techniques for studying biointerfaces. 


\title{
Label-free Detection of Human Prostate-Specific Antigen (hPSA) Using Film Bulk Acoustic Resonators (FBARs)
}

\author{
Zhao et al
}

Figures
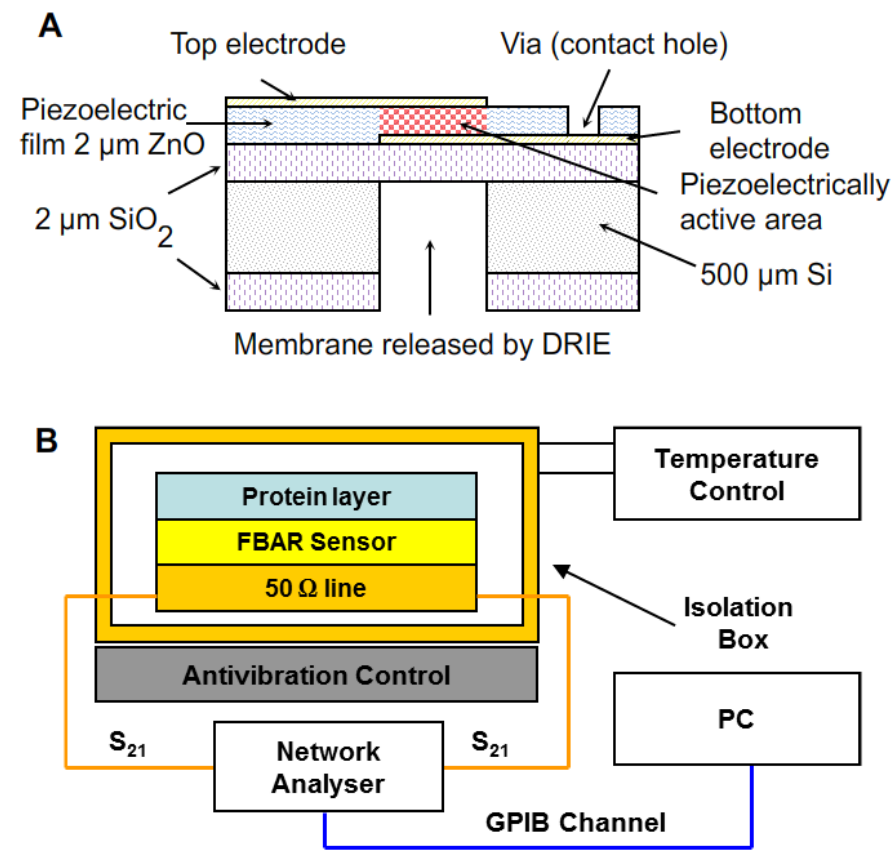

Figure 1. (A) Schematic cross-sectional view of the FBAR device used in the experiments (not to scale). The active sensing area is where the top and bottom electrodes overlap. (B) Schematic diagram (not to scale) of the experimental setup. 


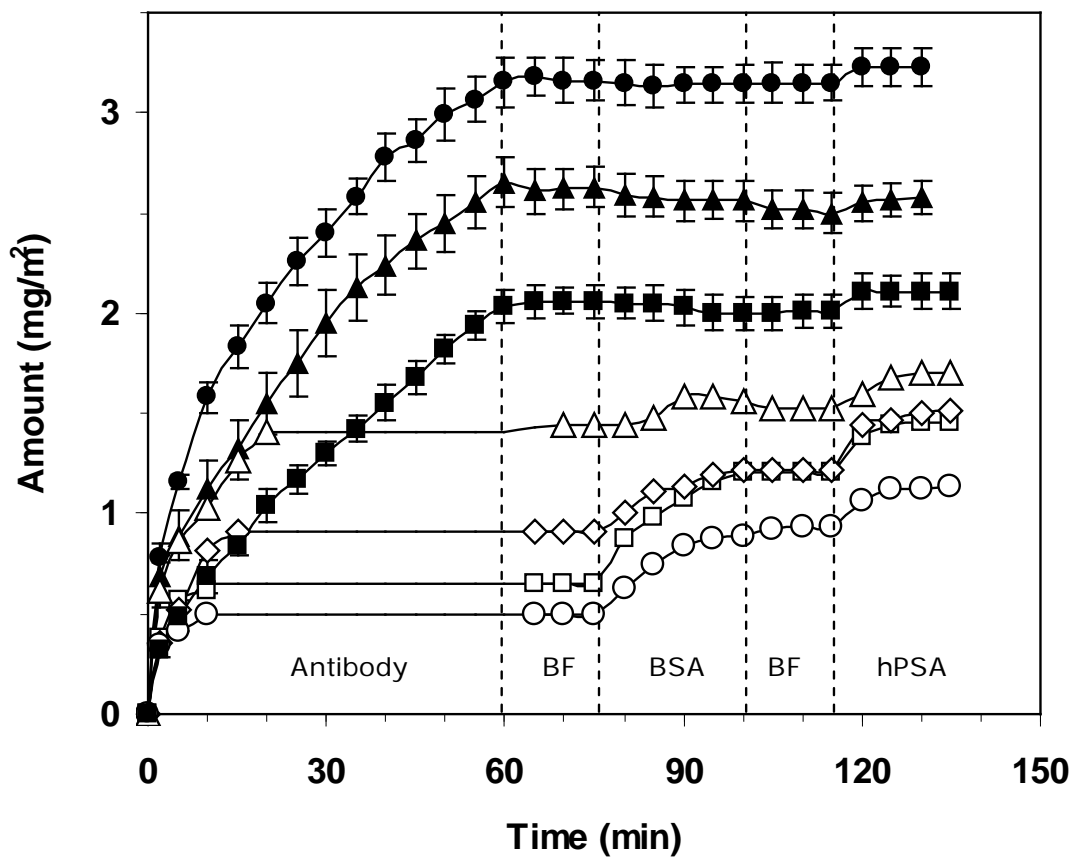

Figure 2. Ellipsometric measurements of Anti-hPSA adsorption at $5(\mathbf{n}, \square, 0, \diamond), 10(\mathbf{\Delta}, \Delta)$ and 20 $(\bullet) \mathrm{mg} / \mathrm{L}$ at the Au/buffer interface followed by buffer wash (BF), BSA (50 mg/L) blocking, buffer wash again and hPSA (5 mg/L) antigen binding. Phosphate buffer at the total ionic strength $(\mathrm{I})=$ $20 \mathrm{mM}$, pH 7 was used throughout the experiments. Adsorption of $5(\square, \circ, \diamond)$ and $10(\Delta) \mathrm{mg} / \mathrm{L}$ were interrupted by replacing with buffer at different times to achieve different amounts of antibody adsorbed at the interface. Each curve was repeated three times. For clarity reason error bars were only shown for the top three curves and the level of the errors for other curves was found similar. 


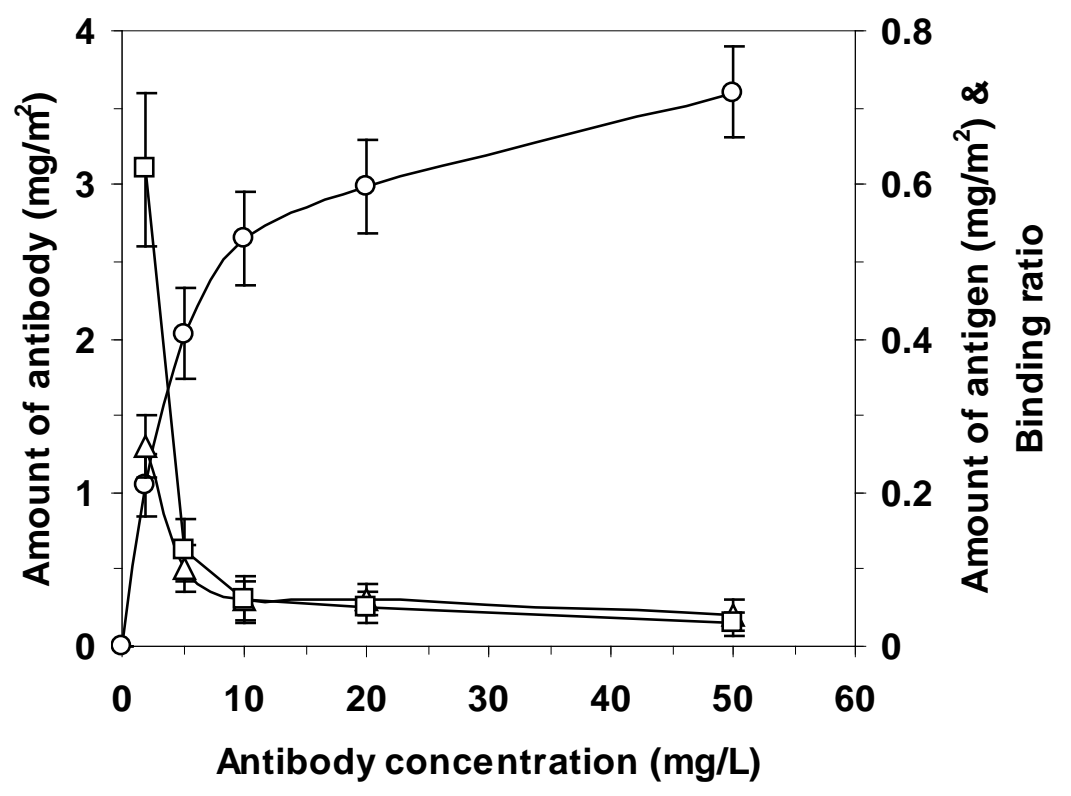

Figure 3. Adsorbed amount of antibody ( $\mathrm{O}$, left axes) at $1 \mathrm{~h}$ and subsequent antigen binding amount ( $\Delta$, right axes) and binding ratio ( $\square$, right axes) against antibody concentration. Lines are for eye guidance. 


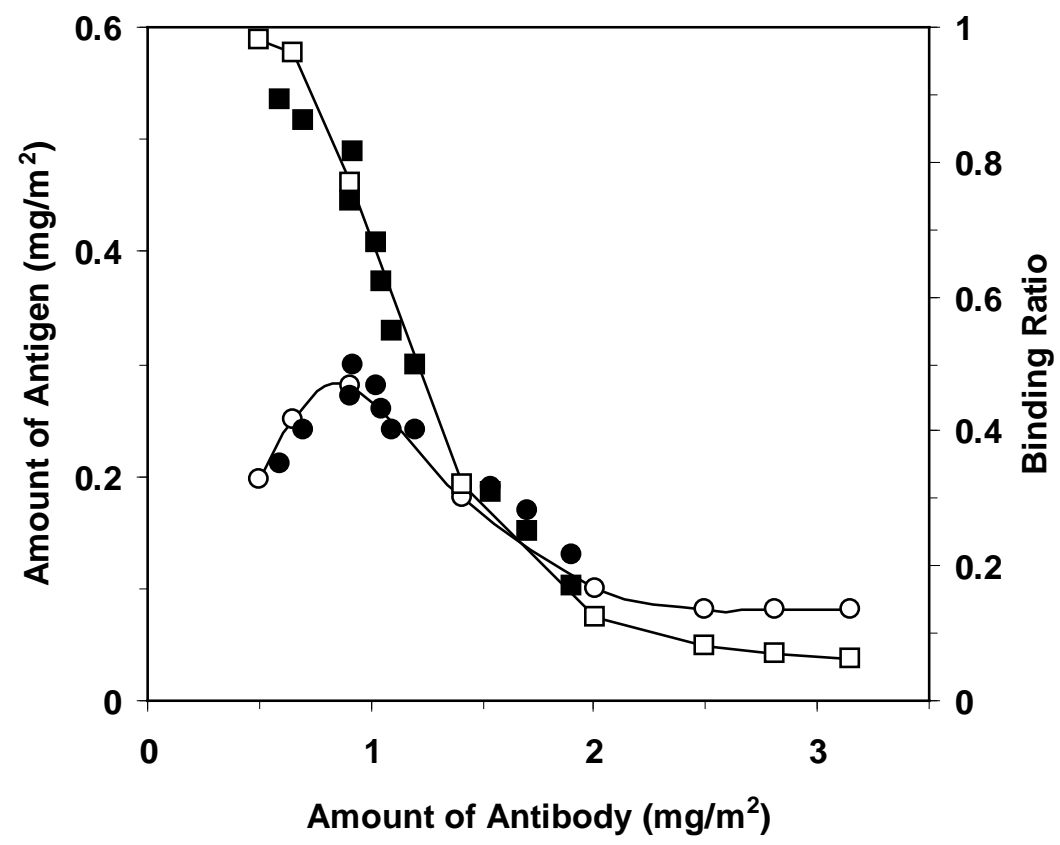

Figure 4. Antigen binding amount ( $\circ$ and $\bullet$, left axes) and binding ratio ( $\square$ and $\mathbf{\square}$, right axes) against the amount of antibody immobilized on gold surface. Data were obtained from $5 \mathrm{~nm}$ thick Au surface ( $($ and $\square)$ and $50 \mathrm{~nm}$ thick Au surface (• and $\mathbf{\square})$ which is the same as FBAR top electrode. Lines are for eye guidance. 

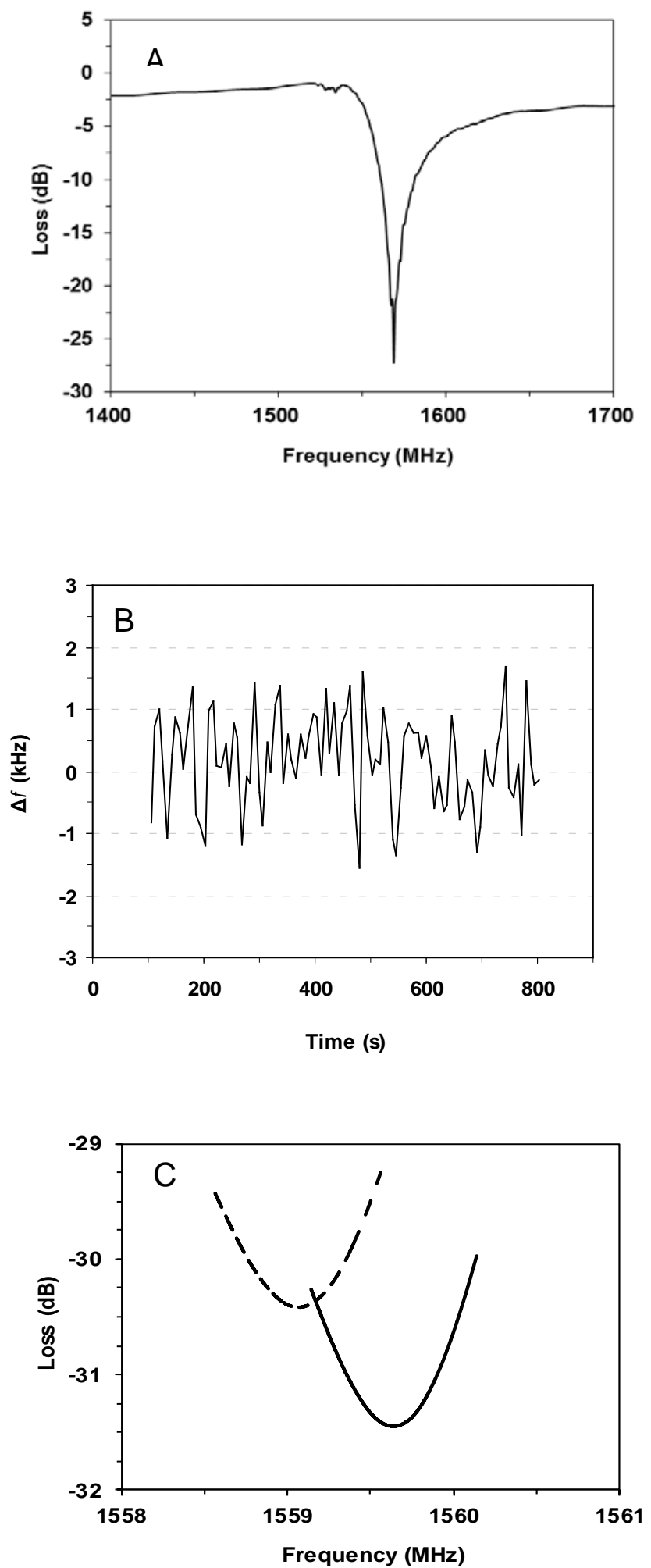

Figure 5. (A), a typical frequency response $\left(S_{21}\right.$ parameter $)$ from fabricated devices. The sharp resonance of the $\mathrm{ZnO}$ film indicates a very high quality factor. (B), the change of resonant frequency with time shows very low frequency indeterminacy (around $3 \mathrm{kHz}$ ). (C), a typical resonant frequency shift-down of $600 \mathrm{kHz}$ after antibody adsorption. The Right curve is the frequency response of the bare FBAR while the left curve is the frequency response after antibody adsorption. 

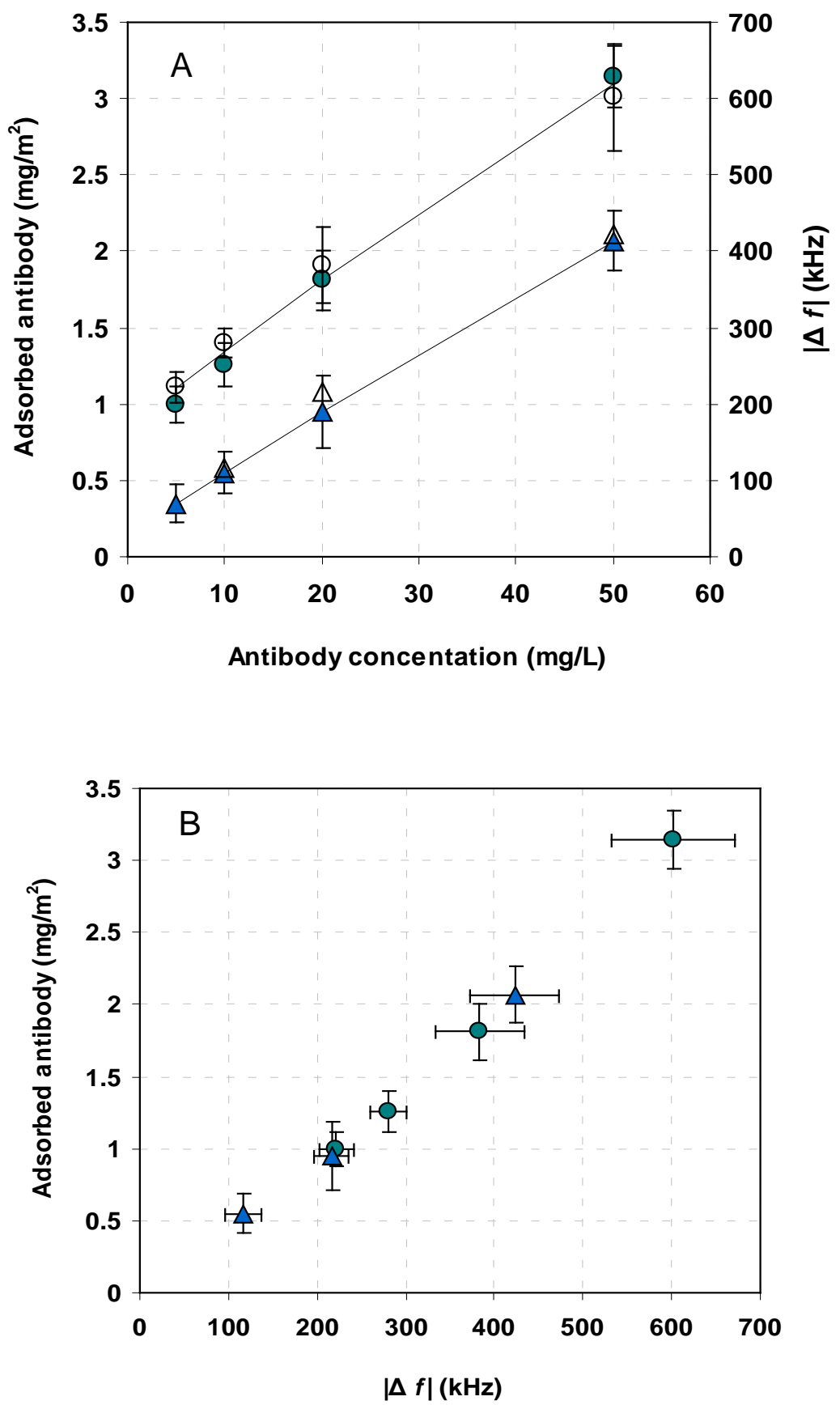

Figure 6. (A), Amount of antibody (left axes) adsorbed on the Au surface at $2(\mathbf{\Delta})$ and $15(\bullet) \min$ at different solution concentrations measured by ellipsometry and the frequency shift-down (right axes) of FBARs resulted from the antibody adsorption for $2 \mathrm{~min}(\Delta)$ and $15 \mathrm{~min}(\mathrm{O})$ at different concentrations. Lines are guide to the eye. (B), Surface adsorbed amount of antibody obtained from ellipsometry plotted against FBAR frequency shift-down. 


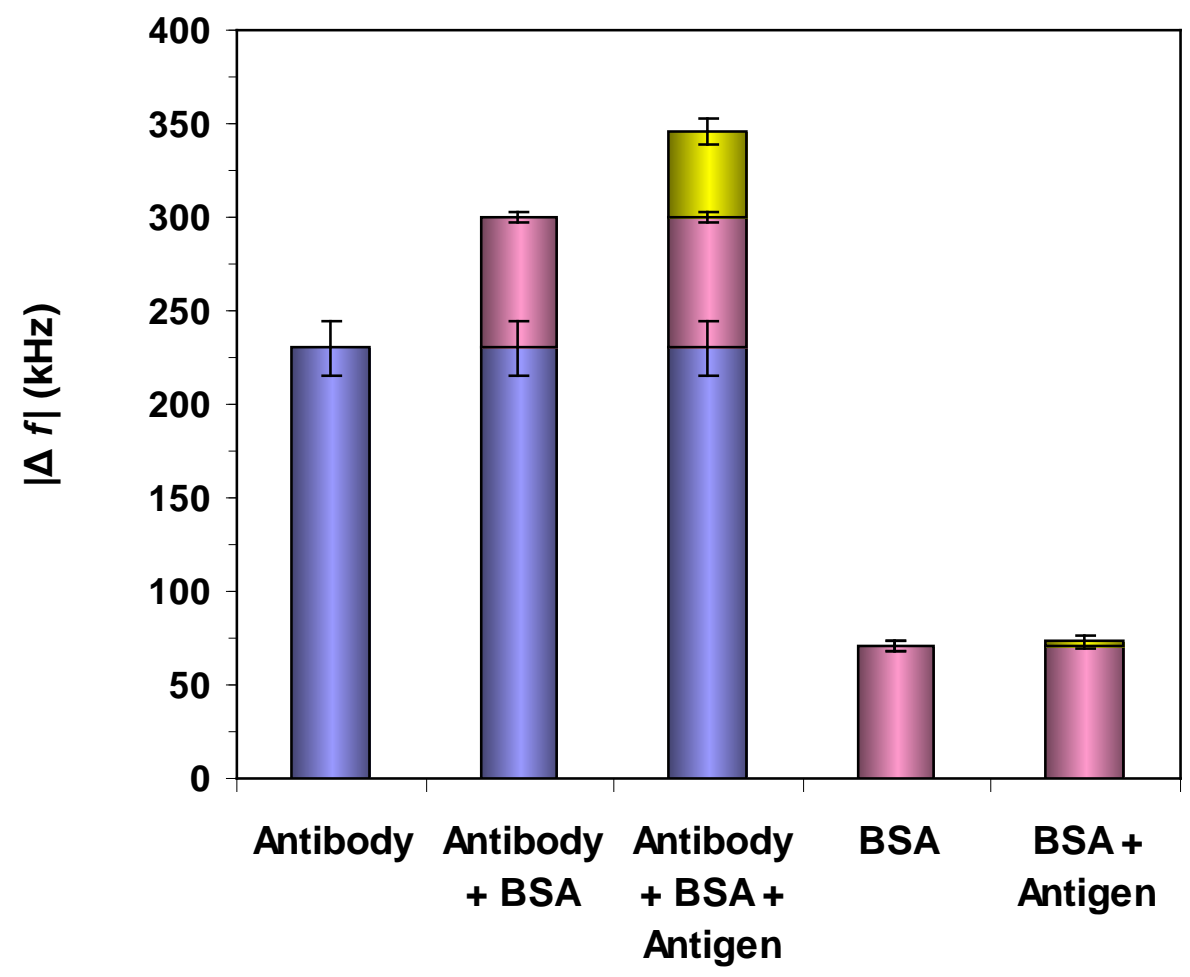

Figure 7. Resonant frequency shifts of FBAR after the adsorption of anti-hPSA antibody (5 mg/L) for $15 \mathrm{~min}$, BSA (50 mg/L) blocking for $25 \mathrm{~min}$ and antigen ( $\mathrm{hPSA}, 5 \mathrm{mg} / \mathrm{L}$ ) binding for $15 \mathrm{~min}$. A control experiment is also shown for the BSA adsorption on electrode surface for $30 \mathrm{~min}$ and followed by antigen adsorption on top of BSA for $15 \mathrm{~min}$. Colour indication: blue for antibody; purple for BSA and yellow for antigen. 